\title{
SULFADOXINE-PYRIMETHAMINE PLUS CHLOROQUINE OR AMODIAQUINE FOR UNCOMPLICATED FALCIPARUM MALARIA: A RANDOMIZED, MULTISITE TRIAL TO GUIDE NATIONAL POLICY IN UGANDA
}

\author{
NATHAN BAKYAITA, GRANT DORSEY, ADOKE YEKA, KRISTIN BANEK, SARAH G. STAEDKE, MOSES R. KAMYA, \\ AMBROSE TALISUNA, FRED KIRONDE, SAM NSOBYA, ALBERT KILIAN, ARTHUR REINGOLD, \\ PHILIP J. ROSENTHAL, AND FRED WABWIRE-MANGEN \\ Ministry of Health, Kampala, Uganda; Department of Medicine, San Francisco General Hospital, University of California, San \\ Francisco, California; Makerere University Medical School, Kampala, Uganda; Division of Epidemiology, School of Public Health, \\ University of California, Berkeley, California; Institute of Public Health, Makerere University, Kampala, Uganda
}

\begin{abstract}
The use of combinations of inexpensive drugs for the treatment of malaria in Africa has been proposed as an interim policy while awaiting the widespread availability of more effective regimens. We compared sulfadoxinepyrimethamine plus chloroquine or amodiaquine in three districts in Uganda. Patients aged 6 months or greater with uncomplicated falciparum malaria were enrolled and randomized to therapy. Safety, tolerability, and efficacy outcomes, adjusted by genotyping, were assessed over 28 days. Of 1,105 patients enrolled, 1,057 (96\%) completed follow-up. For children less than 5 years old, the risk of clinical treatment failure adjusted by genotyping at the three sites ranged from $34 \%$ to $67 \%$ with chloroquine plus sulfadoxine-pyrimethamine and from $13 \%$ to $35 \%$ with amodiaquine plus sulfadoxine-pyrimethamine (risk differences $21-32 \%, P<0.0001$ at all sites). Serious adverse events were uncommon with both regimens. The risk of treatment failure with chloroquine plus sulfadoxine-pyrimethamine, the current standard in Uganda, was unacceptably high. Amodiaquine plus sulfadoxine-pyrimethamine was significantly more efficacious; however, existing levels of resistance raises concern about the useful therapeutic life-span of this regimen.
\end{abstract}

\section{INTRODUCTION}

Malaria remains one of the most serious global health problems. In sub-Saharan Africa, the spread of resistance to the widely available and inexpensive drugs chloroquine (CQ) and sulfadoxine-pyrimethamine (SP) poses a major threat to targets to halve the malaria burden by the year $2010 .{ }^{1}$ Indeed, the continued use of ineffective drugs has been linked to increased malarial incidence and mortality. ${ }^{2,3}$ Few effective, affordable antimalarial agents are currently available, and appropriate alternatives to $\mathrm{CQ}$ and SP have been difficult to identify. One alternative regimen, amodiaquine (AQ) plus SP, has shown surprisingly good efficacy in Uganda, ${ }^{4-6}$ although concerns persist about its effectiveness in different geographical settings and over time. Many authorities have strongly advocated the use of combination antimalarial regimens in Africa, particularly artemisinin-based combination therapy (ACT), ${ }^{7}$ but implementation of this recommendation is complicated by limited clinical experience with newer regimens and the higher cost of artemisinins.

To reevaluate antimalarial treatment policies, in 1997 Uganda's Malaria Control Program (MCP), with the support of the East African Network for Monitoring Antimalarial Treatment (EANMAT), established seven sentinel sites representative of the various geographical, epidemiologic, and ecological strata of malaria in Uganda. Studies at 6 of these sites in 1998-1999 demonstrated median clinical failure rates in children aged 6-59 months of $28 \%$ for CQ (range, $10-46 \%$ ) and $10 \%$ for SP (range, $0-18 \%$ ). ${ }^{8}$ However, these studies were limited by small sample sizes, noncomparative study designs, and a 14-day follow-up period, which may have significantly underestimated the true risk of treatment failure. ${ }^{4}$ In June 2000, the combination of CQ + SP was selected to replace $\mathrm{CQ}$ as first-line treatment of uncomplicated malaria, although limited efficacy data for this combination were available. In Kampala, CQ + SP was subsequently shown to be more efficacious than SP monotherapy but inferior to AQ + SP after 14 days of follow-up. ${ }^{5}$ The choice of CQ + SP was not ideal, as it consists of two drugs that are clearly failing, ${ }^{7}$ but its implementation was necessitated by the urgent need to replace CQ monotherapy and a shortage of efficacy data for other therapies, especially from Uganda. In this regard, immediate interest focused on the possibility of replacing CQ + $\mathrm{SP}$ with AQ + SP, which is currently available and has shown surprisingly strong efficacy in three studies in Kampala ${ }^{4-6}$ but had not been studied in other regions of Uganda.

Here, we describe the establishment of an expanded national program to monitor antimalarial drug resistance in Uganda based on the EANMAT model and report the results of a multisite randomized clinical trial comparing the efficacy, safety, and tolerability of CQ + SP versus AQ + SP. We show that multisite randomized clinical trials, with long-term follow-up, molecular genotyping, and full safety assessment, can be conducted within a Ministry of Health framework to generate the best available evidence to guide policy decisions. We also discuss the implications of our results for antimalarial drug policy in Uganda.

\section{MATERIALS AND METHODS}

Organizational structure and study sites. The Uganda Malaria Surveillance Project (UMSP) was formed as a collaborative effort between the Ugandan Ministry of Health $(\mathrm{MOH})$ and academic researchers to build local capacity and implement a multisite surveillance system to evaluate the comparative efficacy, safety, and tolerability of antimalarial therapies for the development of evidence-based treatment policy. The UMSP organization consists of a core facility, which provides training, logistical support, data management and quality control, and study teams working at seven sentinel sites around Uganda. Sentinel sites, which are based within public health facilities, were previously selected to be representative of the range of ecological and epidemiologic conditions by the Ugandan MCP in collaboration with EANMAT. ${ }^{9}$ In this report we present results from our first three studies, which were conducted between December 2002 
and June 2003 at sites in Kanungu, Kyenjojo, and Mubende districts. All sites are rural, with two annual peaks in rainfall and medium to high malaria transmission intensity (Ugandan $\mathrm{MOH}$, unpublished data). A common protocol was approved by the Ugandan National Council for Science and Technology, and institutional review boards at the University of California, San Francisco, and the University of California, Berkeley.

Recruitment. All patients who presented to the sentinel site health centers with symptoms suggestive of malaria and a positive screening thick blood smear were referred to study physicians for further evaluation. Consecutive patients were screened for the following selection criteria: 1) age 6 months or greater; 2) history of fever in the last 24 hours or axillary temperature greater or equal to $37.5^{\circ} \mathrm{C} ; 3$ ) no history of serious side effects to study medications; 4) no evidence of a concomitant febrile illness; 5) provision of informed consent; 6) ability to participate in 28-day follow-up; 7) no history of treatment with an antifolate or AQ during the previous week; 8) absence of pregnancy based on history of last menstrual period; and 9) no danger signs (prostration, inability to drink, recent convulsion, persistent vomiting) or evidence of severe malaria. ${ }^{10}$ The final selection criteria, including: 10) Plasmodium falciparum monoinfection; 11) parasite density $2000 / \mu \mathrm{L}$ to $200,000 / \mu \mathrm{L}$; and 12 ) hemoglobin $\geq 5.0 \mathrm{~g} / \mathrm{dL}$, were assessed after enrollment. Because laboratory results were not available until the following day, a patient could be excluded after randomization. Patients with persistent vomiting of study medications on Day 0 were also excluded.

Baseline evaluation, randomization, and treatment allocation. At enrollment, patients or their parents/guardians were questioned regarding prior antimalarial therapy, use of other medications, and common symptoms. Temperature (axillary) and weight were measured, and a physical examination was performed. Blood was obtained by fingerprick for thick and thin blood smears, hemoglobin measurement, and storage on filter paper for molecular analysis. Patients were randomly assigned to receive one of two oral treatments: CQ plus SP or AQ plus SP. Medications were dosed as follows: chloroquine (Cosmoquin, Cosmos, $150 \mathrm{mg}$ base tablets, $25 \mathrm{mg} / \mathrm{kg}$, maximum dose $1500 \mathrm{mg}$ base) $10 \mathrm{mg} / \mathrm{kg}$ on Days 0 and 1 , and 5 $\mathrm{mg} / \mathrm{kg}$ on Day 2; SP (Fansidar, Roche, $500 \mathrm{mg} / 25 \mathrm{mg}$ tablets, $25 \mathrm{mg} / \mathrm{kg}$ of sulfadoxine and $1.25 \mathrm{mg} / \mathrm{kg}$ of pyrimethamine, maximum dose 3 tablets) as a single dose; and amodiaquine (Camoquin, Parke-Davis, $200 \mathrm{mg}$ base tablets, $25 \mathrm{mg} / \mathrm{kg}$, maximum dose $1500 \mathrm{mg}$ base) $10 \mathrm{mg} / \mathrm{kg}$ on Days 0 and 1 , and $5 \mathrm{mg} / \mathrm{kg}$ on Day 2.

Randomization codes were computer-generated for two age groups (6-59 months and 5 years or older) by an off-site investigator. Randomization codes were provided to a study nurse who was responsible for treatment allocation. Patients were not informed of their treatment regimen, and all other study personnel were blinded to the treatment assignments. Study medications were administered by the study nurse according to weight-based guidelines for administration of fractions of tablets modified from World Health Organization (WHO) recommendations. ${ }^{11}$ Patients were directly observed for 30 minutes after treatment and the dose was readministered if vomiting occurred. Patients who vomited persistently were excluded from the study and referred for treatment with parenteral quinine. All patients were provided with a 3-day supply of acetaminophen for treatment of febrile symptoms.
Children with hemoglobin levels less than $10.0 \mathrm{~g} / \mathrm{dL}$ were treated according to Integrated Management of Childhood Illness guidelines with ferrous sulfate for 14 days and given antihelminthic treatment if they were more than 1 year of age and had not been treated in the previous 6 months.

Follow-up procedures. Patients were asked to return for follow-up visits on days 1, 2, 3, 7, 14, 21, 28, and any other day when they felt ill. On each visit, a complete history was taken and physical examination performed. Blood was obtained by fingerprick on all follow-up days, except Day 1, for thick blood smears and storage on filter paper. Hemoglobin measurement was repeated on Day 28 or the day of clinical treatment failure. If patients did not return for follow-up, they were visited at home.

Laboratory procedures. Initial screening blood smears were stained with $10 \%$ Giemsa for 10 minutes. Thick and thin blood smears used in the study were stained with $2 \%$ Giemsa for 30 minutes. Parasite densities were determined from thick blood smears by counting the number of asexual parasites per 200 WBCs (or per 500, if the count was less than 10 parasites/ $200 \mathrm{WBCs}$ ) and calculating parasites/microliter assuming a WBC count of $8,000 / \mu \mathrm{L}$. A smear was considered negative if no parasites were seen after review of 100 high-powered fields. Gametocytemia was also assessed from thick blood smears. Thin blood smears were reviewed to detect nonfalciparum infections. Hemoglobin measurements were made using a portable spectrophotometer (HemoCue, Anglholm, Sweden). Filter paper blood samples collected at enrollment and on the day of clinical or parasitological failure were assessed with molecular genotyping performed at University of California, San Francisco, to distinguish recrudescent and new infections. For patients with recurrent parasitemia after Day 4, samples from enrollment and the time of failure were analyzed for polymorphisms in merozoite surface protein-2 (MSP-2), according to previously published methods. ${ }^{12}$ Outcomes were classified as recrudescences (true treatment failure) if all MSP-2 alleles present on the day of recurrence were present at enrollment and as a new infection if this was not the case. Samples with unsuccessful genotyping (1\%) were classified as recrudescences. The potential limitation of this classification is the possibility that posttreatment samples truly do contain both recrudescent parasites and newly infecting strains (mixed results) thus underestimating true failure.

Quality control. Study sites were visited every 2 weeks to monitor study procedures. After each site visit, blood slides were brought to the core facility to assess slide quality and accuracy of microscopy readings. At the beginning of each study, all slides were re-read by senior laboratory technicians. Once error rates were less than $10 \%$, a random sample of $10 \%$ of slides was reviewed. A third reviewer reconciled discordant results.

Adverse event reporting. At each follow-up visit, patients were assessed for any new or worsening event. An adverse event was defined as any untoward medical occurrence, irrespective of its suspected relationship to the study medications as per International Conference of Harmonization (ICH) guidelines (Guidance for Industry Good Clinical Practice: Consolidated Guidance [ICH E6], April 1996). All events were graded by severity (none, mild, moderate, severe, lifethreatening). Relationship to the study medications (none, unlikely, possible, probable, or definite) was recorded for events of moderate or greater severity according to a system 
developed using guidelines from the World Health Organization (Toxicity Grading Scale for Determining the Severity of Adverse Events) and the National Institutes of Health, Division of Microbiology and Infectious Diseases (Pediatric Toxicity Tables, May 2001). A serious adverse event was defined as any adverse experience that resulted in death; lifethreatening experience; hospitalization; persistent or significant disability or incapacity; or specific medical or surgical intervention to prevent serious outcome.

Assessment of treatment response. Day 28 treatment outcomes were classified according to WHO guidelines for areas of intense transmission as adequate clinical and parasitological response (ACPR), early treatment failure (ETF), late clinical failure (LCF), and late parasitological failure (LPF). ${ }^{10}$ The criteria for LCF was modified to include history of fever within the previous 24 hours on Days 4-28 with parasitemia. The classification of LPF, defined as parasitemia in the absence of fever on day 28, was made at the end of study followup. Patients classified as treatment failures (ETF, LCF, LPF) were given rescue therapy with quinine. Clinical outcomes were stratified into success $(\mathrm{ACPR} / \mathrm{LPF})$ or failure $(\mathrm{ETF} /$ LCF). Parasitological outcomes were stratified into success $(\mathrm{ACPR})$ or failure $(\mathrm{ETF} / \mathrm{LCF} / \mathrm{LPF})$. All treatment failures were treated with quinine $(10 \mathrm{mg} / \mathrm{kg}$ three times daily for 7 days). Patients were not assigned an outcome if any of the following occurred: 1) use of antimalarial drugs outside of the study protocol; 2) parasitemia in the presence of a febrile, nonmalarial illness diagnosed by the study physician; 3) withdrawal of consent; and 4) loss to follow-up.

Statistical analysis. Sample size was calculated for each site to test the hypothesis that treatment with AQ + SP would decrease the risk of Day 28 clinical failure adjusted for genotyping compared with CQ + SP (two-sided $\alpha$ of 0.05 and power of $80 \%$ ). The risk of treatment failure was estimated to be $15 \%$ for $\mathrm{CQ}+\mathrm{SP}$ and $5 \%$ for $\mathrm{AQ}+\mathrm{SP}$. Allowing for $10 \%$ exclusion after enrollment, the samples sizes per group were 176 for each site.

Data were double-entered (EpiInfo 6.04, Centers for Disease Control and Prevention, Atlanta, GA), verified, and analyzed using SPSS version 10.0 (SPSS, Inc., Chicago, IL) and Stata version 7.0 (Stata Corporation, College Station, TX). Efficacy data were evaluated using a per-protocol analysis, which only included patients with treatment outcomes. An age-stratified analysis for patients less than 5 years of age and patients 5 years or older was planned. Parasite densities were normalized using logarithmic transformation. Categorical variables were compared using $\chi^{2}$ or Fisher's exact test, and continuous variables were compared using an independent samples $t$ test. The primary efficacy outcomes were 28-day clinical and parasitological failure risks both unadjusted and adjusted by genotyping. Risks of clinical and parasitological treatment failure unadjusted by genotyping were expressed as simple proportions. Risks of clinical and parasitological treatment failure after adjustment by genotyping were estimated using Kaplan-Meier survival analysis techniques in accordance with the new WHO protocol. ${ }^{10}$ With survival analysis, data were censored for new infections. Hypothesis testing was made using risk differences, exact $95 \%$ confidence intervals, and $P$ values. Secondary outcomes included clinical outcomes at Day 14, fever clearance, parasite clearance, change in hemoglobin levels between Day 0 and the last day of follow-up, presence of gametocytes during follow-up, and the incidence of adverse events. A $P$ value (two-tailed) of less than 0.05 was considered statistically significant.

Role of the funding source. The funding source played no role in protocol development, data collection, data analysis, data interpretation, or in the writing of the report.

\section{RESULTS}

Patient characteristics. Of 1,973 patients referred for evaluation at our 3 sites during the study period, 763 were excluded during screening and 105 were excluded after treatment assignment but before enrollment (Figure 1). Reasons for exclusion after treatment assignment included non-falciparum malaria infection $(N=34)$, parasite density $<2,000 / \mu \mathrm{L}(N=$ $26)$ or $>200,000 / \mu \mathrm{L}(N=34)$, hemoglobin $<5.0 \mathrm{~g} / \mathrm{dL}(N=$ $2)$, persistent vomiting of study medications on Day $0(N=$ $7)$, and inappropriate consent $(N=2)$. Of the 1,105 patients enrolled, 1057 (96\%) were assigned efficacy outcomes. Reasons for exclusion after enrollment included loss to follow-up $(N=25)$, withdrawal of consent $(N=12)$, use of antimalarial medications outside of the study protocol $(N=10)$, and development of a concomitant febrile illness that interfered with classification of treatment outcome $(N=1)$. In addition, all patients screened $(N=221)$ and enrolled $(N=124)$ during a 1-month period from one site (Mubende) were excluded due to a systematic error in the classification and management of LPFs. Due to a misunderstanding about the new WHO classification system, patients with asymptomatic parasitemia after Day 3 but before Day 28 were classified as LPFs and treated with quinine. For this reason, we were unable to assess whether these patients would have gone on to become late clinical failures, late parasitological failures, or adequate parasitological and clinical responses at the completion of the full 28-day follow-up period. To best prevent the introduction of bias in our results, we decided to exclude the entire cohort of patients enrolled at this site during the 1 -month period when the error occurred. Among patients with treatment outcomes, there were no statistically significant differences in baseline characteristics between the two treatment groups at each site. However, there were statistically significant differences between the sites for age distribution, prior use of antimalarials, mean temperature, mean hemoglobin, and the presence of gametocytes (Table 1).

Treatment efficacy. The 1,057 patients with efficacy outcomes were stratified by age (age less than 5 years versus 5 years or older). Considering all sites, in children less than 5 years old, the risk of early treatment failure was higher with $\mathrm{CQ}+\mathrm{SP}$ compared with $\mathrm{AQ}+\mathrm{SP}$, but this did not reach statistical significance ( $7 \%$ versus $4 \%, P=0.09$ ). In patients 5 years or older, early treatment failure occurred in only 1 of 159 patients receiving $\mathrm{CQ}+\mathrm{SP}$ and 1 of 161 patients receiving $\mathrm{AQ}+\mathrm{SP}$. At Day 14, CQ + SP was significantly inferior to $\mathrm{AQ}+\mathrm{SP}$ in children under 5 years of age, considering both clinical failure unadjusted by genotyping (34\% versus $10 \%$, risk difference $24 \%, 95 \% \mathrm{CI}: 18-29 \%, P<0.0001)$ and clinical failure after adjustment by genotyping (22\% versus $8 \%$, risk difference $14 \%, 95 \%$ CI: 4-24\%, $P=0.005)$. However, limiting follow-up to 14 days seriously underestimated the risk of clinical treatment failure in both treatment groups. A similar trend was observed for older patients (data not shown).

Considering 28-day outcomes at all sites in patients less than 5 years of age, the risk of clinical failure unadjusted by 


\section{Kyenjojo}

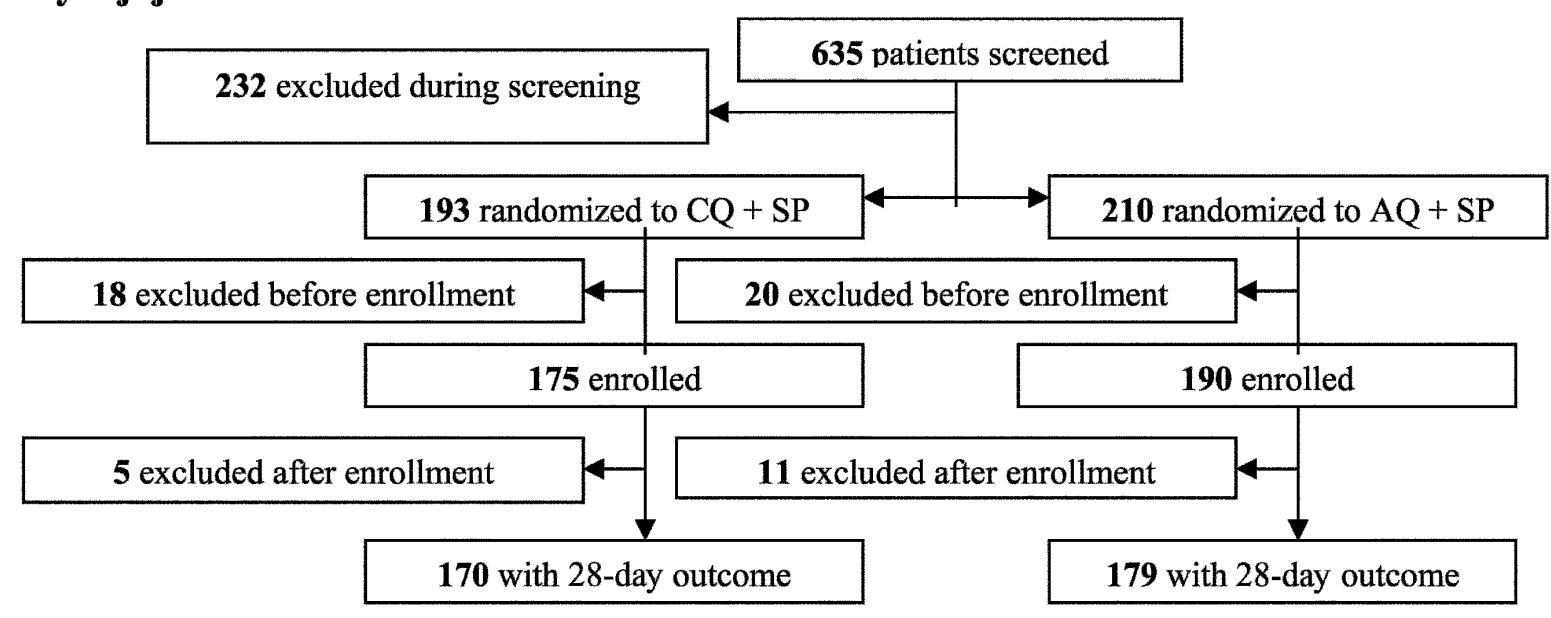

\section{Mubende}

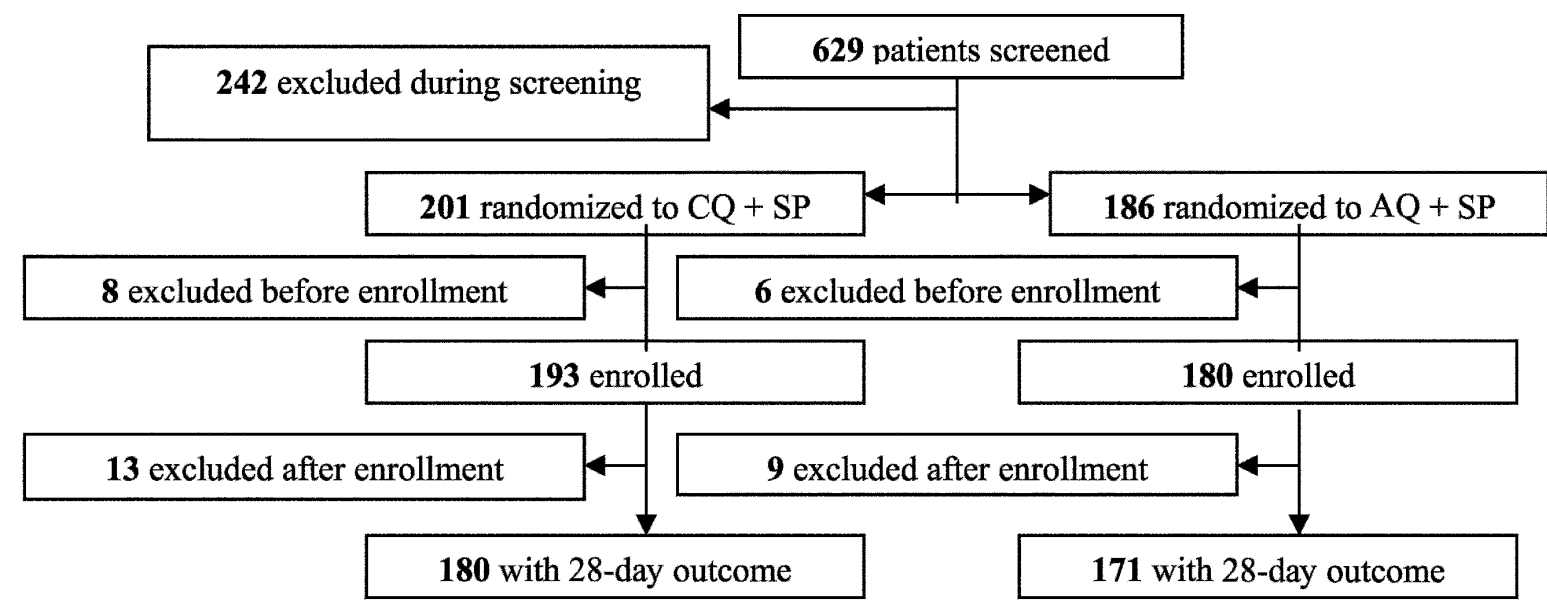

\section{Kanungu}

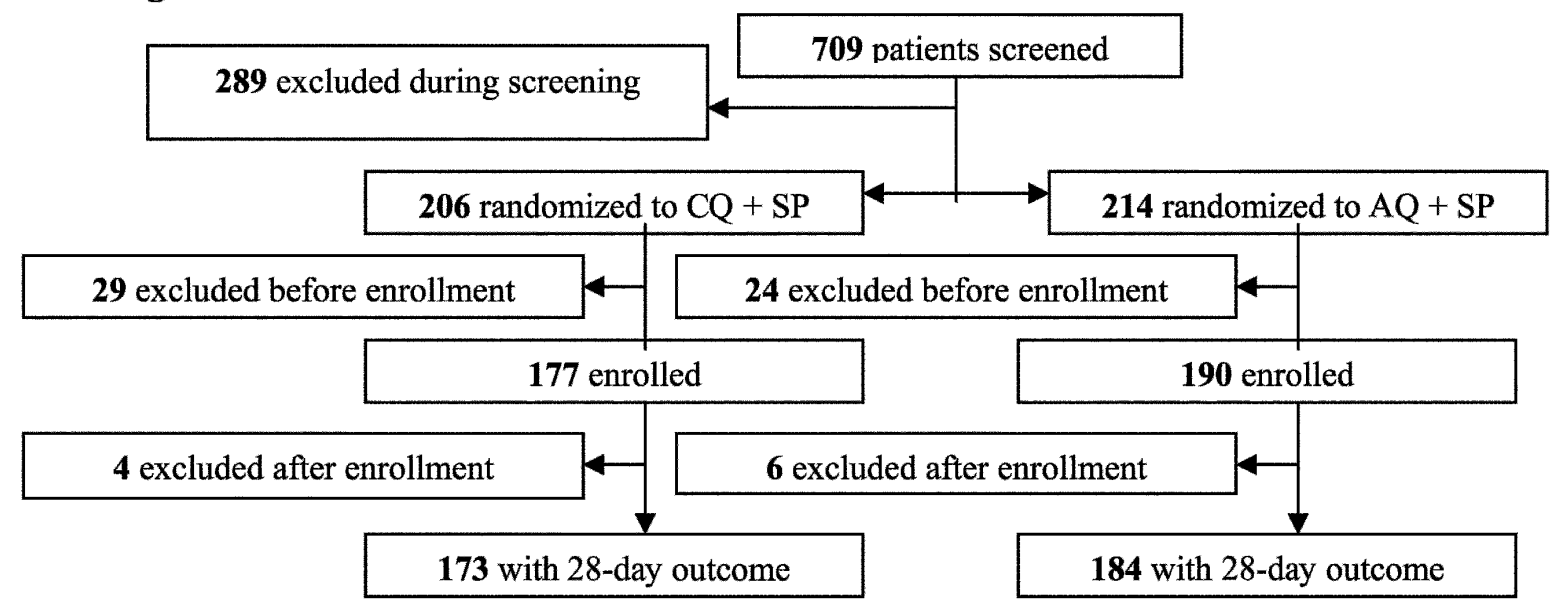

FIGURE 1. Trial profile.

genotyping ranged from $70 \%$ to $86 \%$ with CQ + SP and $35 \%$ to $53 \%$ with $\mathrm{AQ}+\mathrm{SP}$ (risk differences $26-35 \%, P<0.0001$ at all sites) (Table 2). After adjustment by genotyping, the risk of clinical treatment failure ranged from $34 \%$ to $67 \%$ with $\mathrm{CQ}+\mathrm{SP}$ and $13 \%$ to $35 \%$ with $\mathrm{AQ}+\mathrm{SP}$ (risk differences $21-32 \%, P \leq 0.001$ at all sites) (Table 2). Notably, despite differences in risks of clinical failure unadjusted and adjusted by genotyping, the benefit of $\mathrm{AQ}+\mathrm{SP}$ over $\mathrm{CQ}+\mathrm{SP}$ was fairly consistent from site to site. Considering parasitological outcomes, risks of failure unadjusted by genotyping ranged from $81 \%$ to $91 \%$ with CQ + SP and $50 \%$ to $59 \%$ with AQ + SP (risk difference $30-35 \%, P \leq 0.001$ at all sites). The risk of 
TABLE 1

Baseline characteristics of patients with efficacy outcomes

\begin{tabular}{|c|c|c|c|c|c|c|}
\hline & \multicolumn{2}{|c|}{ Kyenjojo } & \multicolumn{2}{|c|}{ Mubende } & \multicolumn{2}{|c|}{ Kanungu } \\
\hline & $\mathrm{CQ}+\mathrm{SP}$ & $\mathrm{AQ}+\mathrm{SP}$ & $\mathrm{CQ}+\mathrm{SP}$ & $\mathrm{AQ}+\mathrm{SP}$ & $\mathrm{CQ}+\mathrm{SP}$ & $\mathrm{AQ}+\mathrm{SP}$ \\
\hline Number & 170 & 179 & 180 & 171 & 173 & 184 \\
\hline Median age in years (IQR) & $3.8(1.6-9.0)$ & $5.0(2.3-11.0)$ & $1.8(1.2-6.0)$ & $1.7(1.0-3.6)$ & $2.0(1.3-3.9)$ & $1.8(1.2-4.3)$ \\
\hline Age less than 5 years, no. (\%) & $96(57 \%)$ & $89(50 \%)$ & $130(72 \%)$ & $139(81 \%)$ & $138(80 \%)$ & $145(79 \%)$ \\
\hline Any antimalarial use previous 2 weeks, no. (\%) & $29(17 \%)$ & $35(20 \%)$ & 0 & $2(1 \%)$ & $71(41 \%)$ & $75(41 \%)$ \\
\hline Mean temperature $\left({ }^{\circ} \mathrm{C}\right)(\mathrm{SD})$ & $37.5(1.4)$ & $37.5(1.3)$ & $38.2(1.0)$ & $38.2(0.9)$ & $37.9(1.3)$ & $37.6(1.3)$ \\
\hline Geometric mean asexual parasites/ $\mu \mathrm{L}$ & 27574 & 24620 & 22275 & 18702 & 23185 & 25068 \\
\hline Mean hemoglobin (g/dL) (SD) & $10.4(2.3)$ & $11.0(2.6)$ & $9.8(2.1)$ & $9.4(2.0)$ & $9.2(2.2)$ & $9.2(2.1)$ \\
\hline Gametocytes present, no. (\%) & $41(24 \%)$ & $41(23 \%)$ & $28(16 \%)$ & $26(15 \%)$ & $7(4 \%)$ & $3(2 \%)$ \\
\hline
\end{tabular}

IQR, intraquartile range.

parasitological failure adjusted by genotyping ranged from $43 \%$ to $73 \%$ with CQ + SP and $14 \%$ to $38 \%$ with AQ + SP (risk difference $29-35 \%, P \leq 0.001$ at all sites) (Table 2).

The risk of treatment failure was considerably lower for patients 5 years of age or greater compared with young children. Considering 28-day outcomes, the risk of clinical failure unadjusted by genotyping ranged from $31 \%$ to $42 \%$ with CQ + SP and $13 \%$ to $31 \%$ with AQ + SP (risk differences 1-29\%, $P \leq 0.05$ at 2 sites). After adjustment by genotyping, the risk of clinical treatment failure ranged from $13 \%$ to $21 \%$ with $\mathrm{CQ}+\mathrm{SP}$ and $3 \%$ to $12 \%$ with $\mathrm{AQ}+\mathrm{SP}$ (risk differences $9-10 \%, P<0.05$ at 1 site). Considering parasitological outcomes, risks of failure unadjusted by genotyping ranged from $52 \%$ to $60 \%$ with CQ + SP and $28 \%$ to $44 \%$ with AQ + SP (risk difference 16-28\%, $P<0.001$ at 1 site). The risk of parasitological failure adjusted by genotyping ranged from $21 \%$ to $40 \%$ with CQ + SP and $10 \%$ to $18 \%$ with AQ + SP (risk difference $10-22 \%, P \leq 0.05$ at 1 site) (Table 2).

Secondary outcomes. Patients treated with AQ + SP had a significantly lower risk of parasitemia on Day 3 at all three sites (Table 3). The proportion of patients with fever on Days 1-3 was lower in the AQ + SP group; however, the results were statistically significant only on Day 2 in Kanungu. Gametocyte carriage during follow-up was lower in the AQ + SP group, but this difference was statistically significant only in Mubende and was of borderline significance in Kanungu. Patients treated with $\mathrm{AQ}+\mathrm{SP}$ had a significantly greater increase in mean hemoglobin at all three sites.

Adverse events. Adverse events, broadly defined as any untoward medical occurrences, occurred commonly. A total of 4,767 events were reported, with 1,007 of 1,057 (95\%) patients completing follow-up experiencing at least 1 event; these events were primarily mild or moderate in severity. A comparison of the most commonly reported adverse events for all study sites combined is presented in Table 4. Considering the occurrence of any adverse event, cough and coryza were significantly more common in the $\mathrm{AQ}+\mathrm{SP}$ group, whereas elevated temperature, subjective fever, and decrease

TABLE 2

Treatment outcomes stratified by age for each study site

\begin{tabular}{|c|c|c|c|c|c|c|c|c|}
\hline & \multicolumn{4}{|c|}{ Age less than 5 years } & \multicolumn{4}{|c|}{ Age 5 years or greater } \\
\hline & $\mathrm{CQ}+\mathrm{SP}$ & $\mathrm{AQ}+\mathrm{SP}$ & $\mathrm{RD}(95 \% \mathrm{CI})$ & $P$ value & $\mathrm{CQ}+\mathrm{SP}$ & $\mathrm{AQ}+\mathrm{SP}$ & $\mathrm{RD}(95 \% \mathrm{CI})$ & $P$ value \\
\hline Kyenjojo & $N=96$ & $N=89$ & & & $N=74$ & $N=90$ & & \\
\hline \multicolumn{9}{|l|}{ Risk of clinical failure } \\
\hline Unadjusted by genotyping* & $70 \%$ & $35 \%$ & $35 \%(21-48 \%)$ & $<0.0001$ & $42 \%$ & $13 \%$ & $29 \%(15-42 \%)$ & 0.0001 \\
\hline Adjusted by genotyping $\dagger$ & $37 \%$ & $14 \%$ & $23 \%(9-37 \%)$ & 0.001 & $13 \%$ & $3 \%$ & $10 \%(1-19 \%)$ & 0.03 \\
\hline \multicolumn{9}{|l|}{ Risk of parasitological failure } \\
\hline Unadjusted by genotyping: & $86 \%$ & $52 \%$ & $35 \%(22-47 \%)$ & $<0.0001$ & $55 \%$ & $28 \%$ & $28 \%(13-42 \%)$ & 0.0004 \\
\hline Adjusted by genotyping $\S$ & $58 \%$ & $24 \%$ & $35 \%(19-51 \%)$ & $<0.0001$ & $21 \%$ & $10 \%$ & $10 \%(-2-22 \%)$ & 0.10 \\
\hline Mubende & $N=130$ & $N=139$ & & & $N=50$ & $N=32$ & & \\
\hline \multicolumn{9}{|l|}{ Risk of clinical failure } \\
\hline Unadjusted by genotyping* & $70 \%$ & $44 \%$ & $26 \%(15-38 \%)$ & $<0.0001$ & $38 \%$ & $16 \%$ & $22 \%(4-41 \%)$ & 0.05 \\
\hline Adjusted by genotyping $\dagger$ & $34 \%$ & $13 \%$ & $21 \%(10-32 \%)$ & 0.0001 & $13 \%$ & $3 \%$ & $10 \%(-2-22 \%)$ & 0.11 \\
\hline \multicolumn{9}{|l|}{ Risk of parasitological failure } \\
\hline Unadjusted by genotyping $\ddagger$ & $81 \%$ & $50 \%$ & $30 \%(20-41 \%)$ & $<0.0001$ & $52 \%$ & $31 \%$ & $21 \%(0-42 \%)$ & 0.07 \\
\hline Adjusted by genotyping§ & $43 \%$ & $14 \%$ & $29 \%(18-41 \%)$ & $<0.0001$ & $26 \%$ & $10 \%$ & $16 \%(-2-33 \%)$ & 0.08 \\
\hline Kanungu & $N=138$ & $N=145$ & & & $N=35$ & $N=39$ & & \\
\hline \multicolumn{9}{|l|}{ Risk of clinical failure } \\
\hline Unadjusted by genotyping* & $86 \%$ & $53 \%$ & $32 \%(22-42 \%)$ & $<0.0001$ & $31 \%$ & $31 \%$ & $1 \%(-20-22 \%)$ & 1.0 \\
\hline Adjusted by genotyping $\dagger$ & $67 \%$ & $35 \%$ & $32 \%(20-45 \%)$ & $<0.0001$ & $21 \%$ & $12 \%$ & $9 \%(-7-25 \%)$ & 0.28 \\
\hline \multicolumn{9}{|l|}{ Risk of parasitological failure } \\
\hline Unadjusted by genotyping $\ddagger$ & $91 \%$ & $59 \%$ & $33 \%(23-42 \%)$ & $<0.0001$ & $60 \%$ & $44 \%$ & $16 \%(-6-39 \%)$ & 0.17 \\
\hline Adjusted by genotyping $\S$ & $73 \%$ & $38 \%$ & $35 \%(23-47 \%)$ & $<0.0001$ & $40 \%$ & $18 \%$ & $22 \%(0-43 \%)$ & 0.05 \\
\hline
\end{tabular}

$\mathrm{RD}$, risk difference.

* Proportion of patients with ETF or LCF

$\dagger$ Risk of ETF or LCF due to recrudescence using Kaplan-Meier product limit formula (LCF due to new infections censored)

$\doteqdot$ Proportion of patients with ETF or LCF or LPF

$\S$ Risk of ETF or LCF/LPF due to recrudescence using Kaplan-Meier product limit formula (LCF/LPF due to new infections censored) 
TABLE 3

Secondary treatment outcomes

\begin{tabular}{|c|c|c|c|c|c|c|c|c|c|}
\hline \multirow[b]{2}{*}{ Outcome } & \multicolumn{3}{|c|}{ Kyenjojo } & \multicolumn{3}{|c|}{ Mubende } & \multicolumn{3}{|c|}{ Kanungu } \\
\hline & $\mathrm{CQ}+\mathrm{SP}$ & $\mathrm{AQ}+\mathrm{SP}$ & $P$ value & $\mathrm{CQ}+\mathrm{SP}$ & $\mathrm{AQ}+\mathrm{SP}$ & $P$ value & $\mathrm{CQ}+\mathrm{SP}$ & $\mathrm{AQ}+\mathrm{SP}$ & $P$ value \\
\hline Proportion with parasitemia on Day 3 & $43 \%$ & $21 \%$ & $<0.0001$ & $43 \%$ & $9 \%$ & $<0.0001$ & $35 \%$ & $19 \%$ & 0.0008 \\
\hline \multicolumn{10}{|l|}{ Proportion with fever* } \\
\hline Day 1 & $85 \%$ & $77 \%$ & 0.06 & $97 \%$ & $99 \%$ & 0.45 & $82 \%$ & $77 \%$ & 0.36 \\
\hline Day 2 & $54 \%$ & $49 \%$ & 0.34 & $73 \%$ & $72 \%$ & 0.90 & $66 \%$ & $50 \%$ & 0.002 \\
\hline Day 3 & $18 \%$ & $20 \%$ & 0.59 & $38 \%$ & $32 \%$ & 0.26 & $32 \%$ & $23 \%$ & 0.08 \\
\hline \multicolumn{10}{|l|}{ Proportion with gametocytes } \\
\hline during follow-up $\dagger$ & $42 \%$ & $36 \%$ & 0.31 & $45 \%$ & $30 \%$ & 0.009 & $30 \%$ & $20 \%$ & 0.06 \\
\hline Change in hemoglobin g/dL (SD) $\ddagger$ & $0.78(1.9)$ & $1.14(1.4)$ & 0.01 & $0.45(1.8)$ & $1.43(1.8)$ & $<0.0001$ & $0.76(1.9)$ & $1.52(2.0)$ & $<0.0001$ \\
\hline
\end{tabular}

* Temperature $\geq 37.5^{\circ} \mathrm{C}$ or history of fever previous 24 hours.

$\dagger$ Only includes those patients with no gametocytes detected on Day 0 .

$\doteqdot$ Hemoglobin on Day 28 or day of failure-Day 0 hemoglobin.

in hemoglobin were significantly more reported in the CQ + SP group. To focus on adverse events most likely related to study medications, patients with treatment failure were removed and analyses restricted to events of moderate or greater severity at least probably related to study medications. In these restricted analyses, the proportions of patients with relevant adverse events were not significantly different between the two treatment groups (Table 4).

A total of 41 serious adverse events were reported in 37 patients, and there were no statistically significant differences in the reporting of serious adverse events between the two treatment groups. Serious adverse events included convulsions $(N=15)$, vomiting $(N=13)$, severe anemia $(N=6)$, weakness $(N=3)$, elevated temperature $(N=2)$, abnormal chest exam $(N=1)$, and leg swelling $(N=1)$. No deaths occurred. Thirty-four of $41(83 \%)$ serious adverse events occurred in patients with clinical treatment failure. Considering the suspected relationship to treatment, 18 were unlikely, 19 were possibly, and 4 (all vomiting) were probably related to study medications.

\section{DISCUSSION}

We conducted a multisite clinical trial in rural Uganda comparing CQ + SP, the standard-of-care for treatment of un- complicated malaria, to $\mathrm{AQ}+\mathrm{SP}$, an inexpensive alternative. Our studies demonstrate that African malaria control programs can perform "state-of-the-art" antimalarial drug efficacy studies at rural sites. Specifically, our studies included randomized, single-blinded comparisons of combination therapies, 28-day follow-up with genotyping to discriminate recrudescence from new infections, comprehensive quality control, and assessment of safety and tolerability. Despite the inherent complexity of conducting a multisite study in rural Africa, we achieved excellent completion rates (>95\%) and collected high-quality data to guide national antimalarial treatment policy.

The World Health Organization (WHO) has provided standardized guidelines for the assessment of antimalarial drug efficacy, designed to collect minimal essential information about treatment response among populations at greatest risk. ${ }^{10}$ These guidelines were recently updated to address previous methodological problems, producing a single standardized protocol adaptable to different transmission levels. Our methods differed slightly from those advocated by the $\mathrm{WHO}^{10}$ in that we directly compared treatment regimens using a single-blinded randomized trial design.

In this study, the risk of clinical and parasitological failure to $\mathrm{CQ}+\mathrm{SP}$ was found to be unacceptably high in three rural

TABLE 4

Comparison of adverse events across all study sites

\begin{tabular}{|c|c|c|c|c|c|c|}
\hline & \multicolumn{3}{|c|}{ Any adverse event } & \multicolumn{3}{|c|}{$\begin{array}{l}\text { Adverse events of moderate or greater } \\
\text { severity and probable/definite relationship* }\end{array}$} \\
\hline & $\mathrm{CQ}+\mathrm{SP}$ & $\mathrm{AQ}+\mathrm{SP}$ & $P$ value & $\mathrm{CQ}+\mathrm{SP}$ & $\mathrm{AQ}+\mathrm{SP}$ & $P$ value \\
\hline \multicolumn{7}{|l|}{ Symptoms } \\
\hline Fever & $360 / 522(69 \%)$ & $298 / 534(56 \%)$ & $<0.0001$ & $31 / 186(17 \%)$ & $52 / 336(15 \%)$ & 0.71 \\
\hline Cough & $249 / 522(48 \%)$ & $306 / 534(57 \%)$ & 0.002 & $2 / 186(1 \%)$ & $6 / 336(2 \%)$ & 0.72 \\
\hline Weakness & $233 / 522(45 \%)$ & $226 / 534(42 \%)$ & 0.46 & 7/186 (4\%) & $20 / 336(6 \%)$ & 0.31 \\
\hline Headache & $88 / 217(41 \%)$ & $91 / 232(39 \%)$ & 0.85 & $3 / 114(3 \%)$ & $1 / 183(1 \%)$ & 0.16 \\
\hline Anorexia & $201 / 522(39 \%)$ & $213 / 534(40 \%)$ & 0.66 & $11 / 186(6 \%)$ & $25 / 336(7 \%)$ & 0.59 \\
\hline Abdominal pain & $77 / 218(35 \%)$ & $91 / 233(39 \%)$ & 0.44 & $2 / 114(2 \%)$ & $0 / 184(0 \%)$ & 0.15 \\
\hline Coryza & $149 / 522(29 \%)$ & $183 / 534(34 \%)$ & 0.05 & $1 / 186(1 \%)$ & $2 / 336(1 \%)$ & 1.0 \\
\hline Vomiting & $151 / 522(29 \%)$ & $144 / 534(27 \%)$ & 0.49 & $9 / 177(5 \%)$ & $7 / 336(2 \%)$ & 0.11 \\
\hline Diarrhea & $135 / 522(26 \%)$ & $140 / 534(26 \%)$ & 0.94 & $1 / 186(1 \%)$ & $3 / 336(1 \%)$ & 1.0 \\
\hline Pruritis & $114 / 522(22 \%)$ & $98 / 533(18 \%)$ & 0.17 & $22 / 186(12 \%)$ & $27 / 335(8 \%)$ & 0.16 \\
\hline \multicolumn{7}{|l|}{ Physical exam findings } \\
\hline Temperature & $218 / 522(42 \%)$ & $159 / 534(30 \%)$ & 0.0001 & $4 / 186(2 \%)$ & $12 / 336(4 \%)$ & 0.43 \\
\hline Abdomen & $77 / 522(15 \%)$ & $83 / 534(16 \%)$ & 0.67 & $0 / 186(0 \%)$ & $0 / 336(0 \%)$ & 1.0 \\
\hline Chest & $58 / 522(11 \%)$ & $67 / 534(13 \%)$ & 0.51 & $0 / 186(0 \%)$ & $2 / 336(1 \%)$ & 0.54 \\
\hline Skin & $42 / 522(8 \%)$ & $45 / 534(8 \%)$ & 0.82 & $4 / 186(2 \%)$ & $8 / 336(2 \%)$ & 1.0 \\
\hline \multicolumn{7}{|l|}{ Laboratory } \\
\hline Decline in hemoglobin $\dagger$ & $114 / 484(24 \%)$ & $63 / 519(12 \%)$ & $<0.0001$ & $5 / 183(3 \%)$ & $10 / 334(3 \%)$ & 1.0 \\
\hline
\end{tabular}

* Excluding clinical treatment failures.

$\dagger$ Any $=$ decline in $\mathrm{Hb}$ to $<10 \mathrm{mg} / \mathrm{dL}$; moderate or greater severity $=$ decline in $\mathrm{Hb}$ to $<9 \mathrm{mg} / \mathrm{dL}$. 
sites around Uganda. Limiting follow-up to 14 days would have seriously underestimated the risk of treatment failure, as most failures occurred between 15 and 28 days after treatment. This result is not surprising, as the long elimination half-life of SP and complex nature of antimalarial drug resistance may delay its clinical presentation. ${ }^{13} \mathrm{AQ}+\mathrm{SP}$ was superior to $\mathrm{CQ}+\mathrm{SP}$ considering both the need for rescue therapy and the risk of true treatment failure (excluding new infections). AQ + SP decreased the need for rescue therapy for symptomatic illness by $31 \%$ in young children and by $20 \%$ in older patients. This difference suggests that replacing CQ + $\mathrm{SP}$ with AQ + SP could decrease the need for repeat therapy of malaria and diminish the risk of progression to severe illness. However, AQ + SP is not an ideal long-term solution, as resistance to both drugs is prevalent and likely increasing. Indeed, the risk of clinical failure with AQ + SP in young children was $35 \%$ at Kanungu, although this was still much lower than that with CQ + SP (67\%).

Our study also offered a rigorous comparison of the safety and tolerability of the two tested regimens. Although, when defined as any untoward medical occurrences, adverse events were very common, drug-related adverse events appeared to be uncommon and generally mild. No significant differences in drug-related adverse events were seen between the two tested regimens. It should be noted that this study was not powered to detect rare, but serious toxicities, which have been described with both SP and $\mathrm{AQ}$, primarily in individuals using the drugs for long-term chemoprophylaxis. ${ }^{14}$

Considering available data, how should the Uganda Malaria Control Program proceed? Clearly, the recent decision to abandon $\mathrm{CQ}+\mathrm{SP}$ as first-line treatment is supported by these results. However, the optimal replacement for $\mathrm{CQ}+\mathrm{SP}$ remains unclear. Artemisinin-based combination therapies (ACT) have been strongly advocated for use in Africa, ${ }^{7}$ but comparative data on the efficacy and safety of available regimens are currently limited in this region. In addition, the high cost and limited availability of these regimens remain barriers to the implementation and sustainability of programs including ACT. ${ }^{15}$ This concern is especially relevant given the emphasis on presumptive treatment of fevers with antimalarials outside of the formal health sector as a cornerstone of malaria control for Africa. In previous studies from Uganda and Rwanda, SP + AS was shown to have a higher risk of treatment failure compared with $\mathrm{AQ}+\mathrm{SP}$, both unadjusted and adjusted for genotyping. ${ }^{4,16}$ Studies comparing CQ + SP, AQ $+\mathrm{SP}$, and $\mathrm{AQ}+\mathrm{AS}$ are currently ongoing in Uganda as part of our national surveillance program. Given the available data and the urgent need to replace $\mathrm{CQ}+\mathrm{SP}$, artemetherlumefantrine was recently chosen as the provisional first-line treatment of uncomplicated malaria in Uganda; however, incorporation of this regimen into the national drug policy, particularly in the home-based management of fever program, will be challenging. AQ + SP could play a role in future policy, either as an interim first-line regimen, pending widespread availability of artemether-lumefantrine, or for use in home-based management of fever. Our results suggest that changing from $\mathrm{CQ}+\mathrm{SP}$ to AQ + SP now could substantially improve therapeutic efficacy. However, the useful therapeutic life of AQ + SP may be limited by resistance to the individual drugs. In Rwanda where AQ + SP is the first-line treatment of uncomplicated malaria, ${ }^{16}$ resistance to this combination at one site, which borders Uganda, was $29 \%$ in 2001 (EANMAT, unpublished data).

The drug efficacy "disaster" in sub-Saharan Africa necessitates ongoing surveillance of antimalarial regimens currently in use and timely evaluation of newer therapies. The WHO has provided basic guidelines to help guide this process, and groups such as EANMAT and WHO/AFRO have helped to coordinate national efforts. Our results demonstrate that clinical trial networks can be established in Africa, allowing for the collection of high-quality data on antimalarial treatment efficacy. Implementation of multisite studies could be done through existing partnerships. Comparative drug efficacy studies using the methodology described here could improve the detection of drug resistance patterns and may uncover dramatic differences in efficacy between available regimens. Ideally, antimalarial drug policy decision-making should be evidence-based, and efforts should be made to maximize the quality of the data collected.

Received May 19, 2004. Accepted for publication October 19, 2004.

Acknowledgments: The authors thank the clinical study team of Joy Bbosa, Nelson Budaka, Oswald Byaruhanga, Moses Musinguzi, Isaac Kigozi, Felix Jurua, Richard Allen, Jonathan Vlahos, Fred Kizito, and Grace Musimenta. We would also like to thank Moses Kigundu, Dan Kyabainze and Regina Nakafero for training the laboratory staff and providing laboratory quality control. We would also like to thank all the health workers at the health centers of Kasambya (Mubende district), Kyenjojo (Kyenjojo district) and Kihihi (Kanungu district), and their respective district administrations, for allowing us to conduct these studies and working alongside the study teams for lengthy periods. We are indebted to the administrative support of Sara Kibirango, Kenneth Mwebaze, and drivers Nuhu Kibampawo, Joshua Sekitoleko, and Marx Dongo who worked tirelessly for the success of these studies. Special thanks goes to John Mpindi the data officer for his tenacity when entering the data and his constant assistance with the data management and Heidi Hopkins who periodically helped with data management and also gave useful comments during the preparation of the manuscript.

Financial support: This work was supported by the Centers for Disease Control/Association Schools of Public Health cooperative agreement, "Malaria Surveillance and Control in Uganda" (SA3569 \& S1932-21/21), and the Department for International Development (DFID).

Authors' addresses: Nathan Bakyaita, Adoke Yeka, Ambrose Talisuna, and Albert Kilian, Ministry of Health/UMSP, P.O. Box 7475, Kampala, Uganda, Telephone: 011-256-41-231563/231569, Fax: 011256-41-540524. Grant Dorsey, Kristin Banek, Sarah G. Staedke, and Philip J. Rosenthal, University of California, San Francisco, Parnassus Avenue, Box 0811, San Francisco, CA 94143, Telephone: 415648-4680, Fax: 415-648-8425. Moses R. Kamya, Fred Kironde, and Sam Nsobya, Makerere University Medical School, P.O. Box 7072, Kampala, Uganda, Telephone: 011-256-41-541188, Fax: 011-256-41540524. Arthur Reingold, University of California, Berkeley School of Public Health, Department of Epidemiology, 140 Warren Hall, MC\#7360, Berkeley, CA 94720, Telephone: 510-642-0327, Fax: 510643-5163. Fred Wabwire-Mangen, Makerere University Institute of Public Health, P.O. Box 7072, Kampala, Uganda, Telephone: 011256-41-532207, Fax: 011-256-41-531807.

Reprint requests: Nathan Bakyaita, Ministry of Health/UMSP, P.O. Box 7475, Kampala, Uganda, Telephone: Office +256 $41231563 / 9$ Ext 249, Cell +256 77 601579, Fax: +256 41 540524, E-mail: nbakyaita@yahoo.com.

\section{REFERENCES}

1. World Health Organization, Position of WHO's Roll Back Malaria Department on malaria treatment policy. http:// www.emro.who.int/rbm. 
2. Korenromp EL, Williams BG, Gouws E, Dye C, Snow RW, 2003. Measurement of trends in childhood malaria mortality in Africa: an assessment of progress toward targets based on verbal autopsy. Lancet Infect Dis 3: 349-358.

3. Trape JF, 2001. The public health impact of chloroquine resistance in Africa. Am J Trop Med Hyg 64: 12-17.

4. Dorsey G, Njama D, Kamya MR, Cattamanchi A, Kyabayinze D, Staedke SG, Gasasira A, Rosenthal PJ, 2002. Sulfadoxine/ pyrimethamine alone or with amodiaquine or artesunate for treatment of uncomplicated malaria: a longitudinal randomised trial. Lancet 360: 2031-2038.

5. Gasasira AF, Dorsey G, Nzarubara B, Staedke SG, Nassali A, Rosenthal PJ, Kamya MR, 2003. Comparative efficacy of aminoquinoline-antifolate combinations for the treatment of uncomplicated falciparum malaria in Kampala, Uganda. Am J Trop Med Hyg 68: 127-132.

6. Staedke SG, Kamya MR, Dorsey G, Gasasira A, Ndeezi G, Charlebois ED, Rosenthal PJ, 2001. Amodiaquine, sulfadoxine/ pyrimethamine, and combination therapy for treatment of uncomplicated falciparum malaria in Kampala, Uganda: a randomised trial. Lancet 358: 368-374.

7. Attaran A, Barnes KI, Curtis C, d'Alessandro U, Fanello CI, Galinski MR, Kokwaro G, Looareesuwan S, Makanga M, Mutabingwa TK, Talisuna A, Francois Trape J, Watkins WM, 2004. WHO, the Global Fund, and medical malpractice in malaria treatment. Lancet 363: 237-240.

8. Kamya MR, Bakyaita NN, Talisuna AO, Were WM, Staedke SG, 2002. Increasing antimalarial drug resistance in Uganda and revision of the national drug policy. Trop Med Int Health 7: 1031-1041.
9. Monitoring antimalarial drug resistance within National Malaria Control Programmes: the EANMAT experience. Trop Med Int Health 6: 891-898.

10. World Health Organization, 2003. Assessment and monitoring of antimalarial drug efficacy for the treatment of uncomplicated falciparum malaria. Geneva: World Health Organization technical document. WHO/HTM/RBM/2003.50.

11. World Health Organization, 1996. Assessment of therapeutic efficacy of antimalarial drugs for uncomplicated falciparum malaria in areas with intense transmission. Geneva: World Health Organization technical document. WHO/MAL/96.1077.

12. Cattamanchi A, Kyabayinze D, Hubbard A, Rosenthal PJ, Dorsey G, 2003. Distinguishing recrudescence from reinfection in a longitudinal antimalarial drug efficacy study: comparison of results based on genotyping of msp-1, msp-2, and glurp. Am J Trop Med Hyg 68: 133-139.

13. White N, 2002. The assessment of antimalarial drug efficacy. Trends Parasitol 18: 458-464.

14. Phillips-Howard PA, West LJ, 1990. Serious adverse drug reactions to pyrimethamine-sulphadoxine, pyrimethaminedapsone and to amodiaquine in Britain. J R Soc Med 83: 82-85.

15. Snow RW, Eckert E, Teklehaimanot A, 2003. Estimating the needs for artesunate-based combination therapy for malaria case-management in Africa. Trends Parasitol 19: 363-369.

16. Rwagacondo CE, Niyitegeka F, Sarushi J, Karema C, Mugisha V, Dujardin JC, Van Overmeir C, van den Ende J, D'Alessandro U, 2003. Efficacy of amodiaquine alone and combined with sulfadoxine-pyrimethamine and of sulfadoxine pyrimethamine combined with artesunate. Am J Trop Med Hyg 68: 743-747. 